\title{
Surgical Outcomes of Pure-Fat Myringoplasty for Small Tympanic Membrane Perforations: A Retrospective Study and Summary of the Literature in the Last 10 Years
}

\author{
Min Kyu Lee ${ }^{\mathbb{D}}$, Tae Min Kim ${ }^{\mathbb{D}}$, Sung Jin Lim ${ }^{\mathbb{D}}$, Dong Heun Park ${ }^{\mathbb{D}}$,

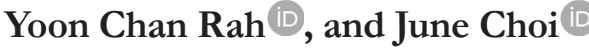 \\ Department of Otorhinolaryngology-Head and Neck Surgery, Korea University Ansan Hospital, Korea University College of Medicine, \\ Ansan, Korea
}

작은 크기의 고막 천공에 대한 순수 지방 고막성형술의 수술 성적:

후향적 연구 및 과거 10 년간의 문헌조사

이민규 · 김태민 · 임성진 · 박동현 · 나윤찬 · 최 준

고려대학교 의과대학 안산병원 이비인후-두경부외과학교실

Received May 1, 2021

Revised July 6,2021

Accepted July 8, 2021

Address for correspondence

June Choi, MD, PhD

Department of Otorhinolaryngology-

Head and Neck Surgery,

Korea University Ansan Hospital,

Korea University

College of Medicine,

123 Jeokgeum-ro, Danwon-gu,

Ansan 15355, Korea

Tel $+82-31-412-5170$

Fax $+82-31-412-5174$

E-mail mednlaw@korea.ac.kr
Background and Objectives Fat myringoplasty is a simple, fast, and effective procedure for repairing tympanic membrane (TM) perforations. The aim of this study is to evaluate the surgical outcomes of pure-fat myringoplasty for small TM perforations at our hospital and review the current knowledge regarding fat myringoplasty, with consideration of the effectiveness of additional substances used in the treatment of small TM perforations.

Subjects and Method We retrospectively studied 41 patients who underwent pure-fat myringoplasty at our hospital from March 2008 through April 2019 and were followed up for at least 2 months thereafter.

Results Of 41 patients, 16 were males and 25 were females, with the mean age of 48 (male, 9-75 years; female, 16-65 years). All perforations were classified as small perforations, ranging from $1 \%$ to $17 \%$ of the TM. The overall success rate was $92.7 \%$, with the TMs of 3 patients (7.3\%) failing to close. Air-bone gap (ABG) decreased in 19 out of 24 patients who underwent both preoperative and postoperative pure tone audiometric examinations. The mean ABG was $4.42 \mathrm{~dB}(\mathrm{n}=24$; paired-t test, $p=0.001 ; 95 \%$ confidence interval, $1.77-7.07)$. The literature review revealed that TM closure success rate of over $80 \%$ was associated with pure-fat myringoplasty, while the TM closure success rate for fat myringoplasty with additional substances was $85 \%$ to $100 \%$.

Conclusion Our study revealed a high TM closure success rate and good hearing outcomes are associated with pure-fat myringoplasty. Pure-fat myringoplasty seems to be sufficient for repairing small TM perforations.

Korean J Otorhinolaryngol-Head Neck Surg 2021;64(10):711-9

Keywords Myringoplasty; Tympanic membrane; Tympanic membrane perforation.

\section{Introduction}

The aim of myringoplasty is to repair of tympanic mem- brane (TM) perforations to improve hearing, reduce susceptibility to middle ear infection, and enable water activities. ${ }^{1,2)}$

Several materials such as fascia, skin, perichondrium, carti-

This is an Open Access article distributed under the terms of the Creative Commons Attribution Non-Commercial License (https://creativecommons.org/licenses/by-nc/4.0) which permits unrestricted non-commercial use, distribution, and reproduction in any medium, provided the original work is properly cited. 
lage, and paper have been used for myringoplasty. ${ }^{3)}$ Among them, fat is a remarkable material, known to have a potential for invigorating the regeneration of the fibrous layer and advancing revascularization. Fat used for graft stimulates vascular endothelial growth factor, transforming growth factor- beta, platelet-derived growth factor, and fibroblast growth factor., ${ }^{1,47)}$

In 1962, Ringenberg was the first to fat as a material for TM perforation repair. ${ }^{3,8}$ Since then, fat myringoplasty has been considered an alternative technique to conventional myringoplasty by many surgeons. ${ }^{3,89}$ Fat myringoplasty is a simpler, faster, and cost-effective office-based procedure that can be conducted under local anesthesia. ${ }^{1,10)}$ Moreover, postauricular fat is easily harvestable in surgical field, and myringoplasty using fat is associated with less morbidity.

In terms of surgical outcomes, several previous publications have reported similar TM closure rates between myringoplasties using fat and those using other materials. ${ }^{911)}$ Therefore, fat is recognized as a suitable myringoplasty material. ${ }^{1,12}$ Recently, some authors have recently suggested substances, such as hyaluronic acid (HA) and platelet-rich plasma (PRP), for use in conjunction with fat to improve success rates associated with fat myringoplasty. ${ }^{11,13-16)}$

This study aimed to evaluate surgical outcomes associated with pure-fat myringoplasty for small TM perforations at our hospital and to review the current knowledge about fat myringoplasty, with consideration of the effectiveness of additional substances.

\section{Subjects and Methods}

\section{Study population and surgical procedures}

We retrospectively reviewed the medical records of 41 patients who were diagnosed with TM perforations and underwent pure-fat myringoplasty procedures between March 2008 and April 2019 at our hospital. All patients satisfied following criteria: 1) central perforation, 2) perforation present for at least 6 months, 3) no evidence of active chronic otitis media, cholesteatoma or retraction pocket formation. Most patients underwent temporal bone computerized tomography preoperatively, with the exception of several patients with TM perforation due to previous ventilation tube insertion. All of patients were followed up for at least 2 postoperative months. The data analyzed in this study included age, sex, size and cause of TM perforation, and the results of pure tone audiometry (PTA). The size of TM perforation was graded according to classification of Saliba's ${ }^{15)}$ subdivision; small perforation (less than $25 \%$ ), medium perforation (more than $25 \%$, less than $50 \%$ ), large perforation (more than $50 \%$, not total), and total perforation ( $100 \%$ or total).

All fat myringoplasty procedures were simple and were performed under sterile conditions by single surgeon (J.C.) using the transcanal approach with a consistent method for all patients. The ear lobe and external auditory canal (EAC) were infiltrated with $1 \%$ lidocaine with 1:100000 epinephrine. The TM perforation margins were trimmed circumferentially under a microscope. Then suitable fat tissue was harvested from the posterior ear lobe. After some pieces of gelfoam were placed in the middle ear, the harvested fat tissue was inserted through the perforation as a champagne cork. After the fat tissue was placed, it was carefully pulled back so that half of the graft was above the perforation to secure eversion of the TM margins. The EAC was packed with some pieces of gelfoam soaked with antibiotic otic solution $(0.3 \%$ ofloxacin solution). An oral antibiotic and otic solution were prescribed for 1 to 2 postoperative weeks.

\section{Assessment of postoperative outcomes and literature summary}

A retrospective review was conducted focusing on audiometric outcomes and the TM closure rate. Additionally, we identified the size, causes, and sites of TM perforation. We used picture archiving and communication system program to accurately measure TM perforation size. The percentage of perforation size to the total TM size was calculated (Fig. 1A).

The mean threshold for PTA was calculated by averaging the hearing thresholds at 4 frequencies $(500,1000,2000$, and $4000 \mathrm{~Hz}$ ). The TM perforation closure rate and change of airbone gap (ABG) were analyzed after at least 2 postoperative months.

\section{Statistical analysis}

All values are shown as mean \pm standard deviation. We used the paired t-test to evaluate whether there was a difference between preoperative hearing status and post-operative hearing outcomes. Also, the Mann-Whitney test and the KruskalWallis test were used to evaluate the difference in postoperative hearing change according to the cause and location of TM perforations. Statistical analyses were performed using R, version 3.5.0 (R Foundation for Statistical Computing, Vienna, Austria). $p$ values less than 0.05 were considered statistically significant. 


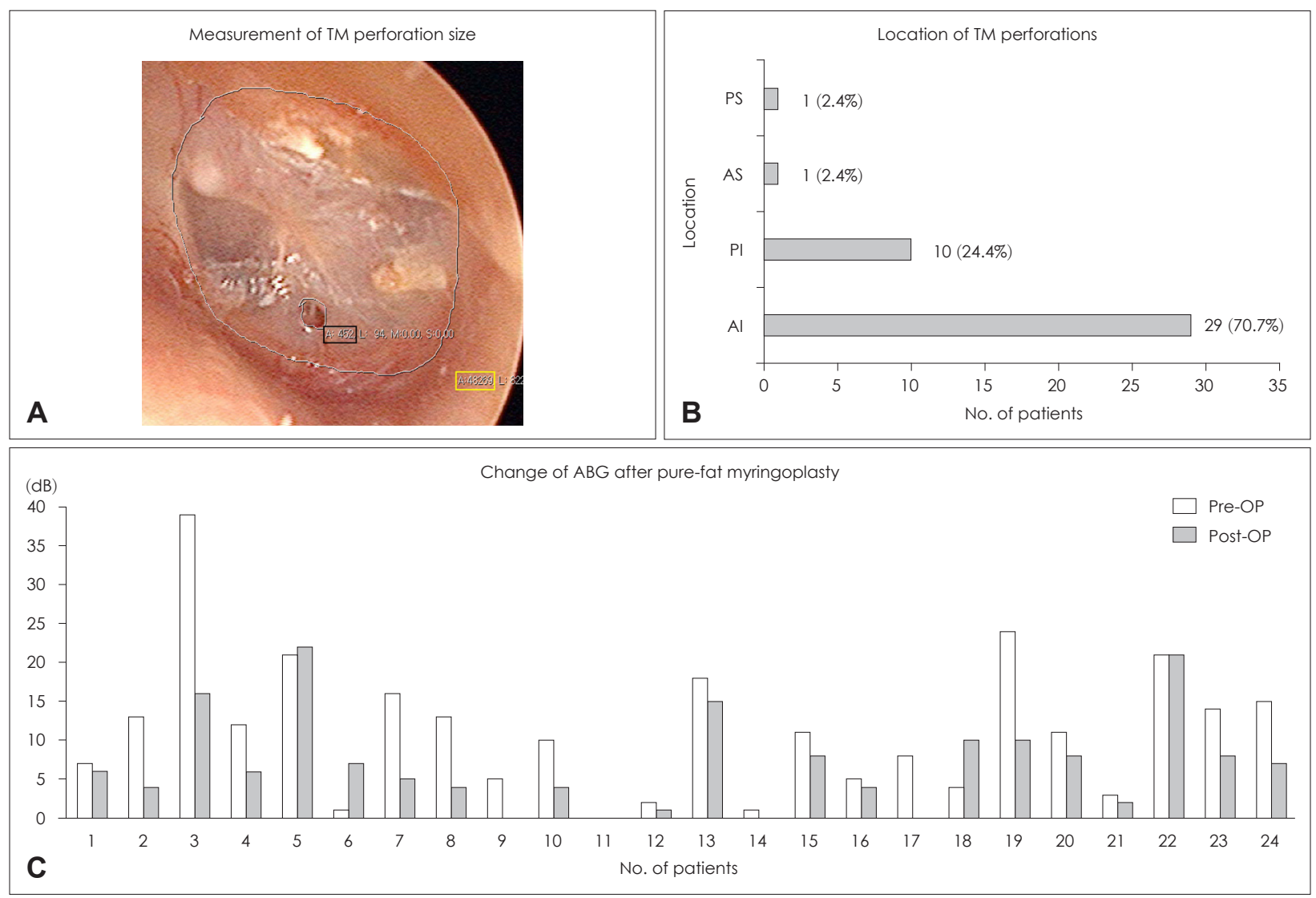

Fig. 1. Results of pure-fat myringoplasty. A: Perforation size was calculated as a ratio of perforation size (black box) and total TM size (yellow box). B: Al site was most common in small perforation. C: The 19 out of 24 patients improved postoperative ABG. TM, tympanic membrane; PS, posterosuperior; AS, anterosuperior; PI, posteroinferior; Al, anteroinferior; ABG, air-bone gap.

Table 1. Causes of tympanic membrane perforations $(n=41)$

\begin{tabular}{lc}
\hline \multicolumn{1}{c}{ Cause } & $\mathrm{n}(\%)$ \\
\hline Previous tympanoplasty & $20(48.8)$ \\
Previous ventilation tube insertion & $7(17.1)$ \\
Chronic inflammation & $6(14.6)$ \\
Trauma & $5(12.2)$ \\
Incidental finding & $3(7.3)$ \\
\hline
\end{tabular}

\section{Ethics statement}

This study was approved by the Institutional Review Board (IRB) of our hospital (IRB number: 2018AS0036).

\section{Results}

\section{Postoperative outcomes of pure-fat myringoplasty}

Forty-one patients were included in the study: 16 males and 25 females. The mean age was 48 (range among males, 9-75 years; range among females, 16-65 years). All patients had small perforation, ranging from $1 \%$ to $17 \%$ of the TM. Anteroinferior TM perforations were the most common (29/41, $70.7 \%$ ), followed by posteroinferior $(10 / 41,24.4 \%)$, anterosu-
Table 2. The closure rate of tympanic membrane after pure-fat myringoplasty

\begin{tabular}{llccc}
\hline Sex & Age $(\mathrm{yr})$ & $\begin{array}{c}\text { Success } \\
(\mathrm{n}=38[92.7 \%])\end{array}$ & $\begin{array}{c}\text { Failure } \\
(\mathrm{n}=3[7.3 \%])\end{array}$ & $\begin{array}{c}\text { Total } \\
(\mathrm{n}=41)\end{array}$ \\
\hline Male & $48(9-75)$ & 15 & 1 & 16 \\
Female & $48(16-65)$ & 23 & 2 & 25 \\
\hline
\end{tabular}

perior (1/41, 2.4\%), and posterosuperior (1/41, 2.4\%) (Fig. 1B).

Previous tympanoplasty was the most common cause of TM perforations $(20 / 41,48.8 \%)$, followed by previous ventilation tube insertion $(7 / 41,17.1 \%)$, chronic inflammation (6/41, $14.6 \%)$, trauma $(5 / 41,12.2 \%)$, and incidental finding $(3 / 41$, $7.3 \%$ ) (Table 1). TM closure was successful in 38 of the 41 patients $(92.7 \%)$ after pure-fat myringoplasty (Table 2$)$. In the 3 cases with TM closure failure, there was no specific difference from the successful group in the perforation location, cause, and size. There were no postoperative complications such as infection in all patients.

Of the 41 patients, 24 patients underwent both preoperative and postoperative PTA, 19 of whom showed postoperative hearing gains. The mean value of hearing changes (preoper- 
ative $\mathrm{ABG}$ - postoperative $\mathrm{ABG}$ ) was $4.42 \mathrm{~dB}$, statistically significant results $(\mathrm{n}=24$; paired t-test; $p=0.001,95 \%$ confidence interval, 1.77-7.07) (Fig. 1C). Even in the group that did not improve hearing, a detailed analysis showed that there was no or slight change in hearing after surgery, or only a relative deterioration within the normal hearing range.

\section{Discussion}

Since Ringenberg ${ }^{8)}$ first introduced ear lobe fat as a graft for TM perforations in 1962, several modifications for improving surgical outcomes have been introduced, including adjunctive graft substances. ${ }^{3)}$ In the present study, we analyzed surgical outcomes of pure-fat myringoplasty for small TM perforations at our hospital, and we summarized surgical results associated with pure-fat myringoplasty and those associated with fat myringoplasty with additional substance in the last 10 years.

TM perforations spontaneously healed via eminent autoregeneration, which is explained by regeneration of the outer squamous epithelium (a centrifugal migration of keratin and proliferation of epithelium) and a formation of a newly created fibrous layer. However, the outer squamous epithelium grows into the inner mucosal side, which inevitably results in a persistent perforation. ${ }^{17,18)}$ In general, it has been suggested that perforation size appears to be the most important factor for determining operative success rates, and the selection of small perforations seems to be ideal for fat myringoplasty, as with conventional myringoplasty. ${ }^{19)}$ In terms of perforation size, closure success rates for large perforations ( $>30 \%$ of TM) have been reported to be significantly lower than those for small perforations. ${ }^{11)}$ Meanwhile, Kim, et al. ${ }^{9)}$ reported that perforation size had no significant bearing on TM closure success rate. Table 3 shows that closure success rates are generally lower in association with larger perforations. Many reports of studies limited to investigations of small perforations report success rate $>80 \%$ like our study. ${ }^{11,20-23)}$

Many researchers have demonstrated appropriate indications or conditions for fat myringoplasty. ${ }^{1,3,10,21)}$ We summarize 2 sets of representative criteria. First, Fiorino and Barbieri ${ }^{1)}$ suggested that 1) the interval since a previous surgery should be $\geq 6$ months; 2 ) perforation of the pars tensa should be no larger than $5 \mathrm{~mm}$; 3 ) there should be non-marginal localization, i.e., involving the annulus or exposing the malleus handle; 4) there should be no calcific plaques or atrophic areas adjacent to the perforation; 5) the mucous layer in the tympanic cavity should appear normal; 6) there should be no acute inflammation; 7) no middle ear discharge in the last 3 months; 8) no evidence of cholesteatoma; 9) no planned ossicular reconstruction; 10) and no major eustachian tube dysfunction. Second, Malafronte and Filosa. ${ }^{10)}$ proposed the following criteria: 1) absence of granulomatous tissue and cholesteatoma, 2) absence of ossicular chain interruption, and 3) perforation size of up to $35 \%$ of the eardrum surface. We believe that although these criteria are not absolute guidelines, they suggest good surgical conditions for fat myringoplasty.

HA and PRP have been chiefly used in fat myringoplasty as additional substances in the last 10 years. HA is a component of the extracellular matrix and is known for being highly biocompatible. Additionally, HA has a potential for stimulating regeneration of the fibrous layer, supports excess keratin formation of the outer epithelial layer, and also promotes a centripetal migration of the outer epithelial layer during TM healing. ${ }^{1824)}$ It is known that liquid form of HA can be used in the middle ear, as its solid polyester form is perfectly reabsorbed within 8 weeks. ${ }^{17,24)}$ Based on these characteristics of HA, some researchers have reported fat myringoplasty with HA to have superior success rates than pure-fat myringoplasty, ${ }^{2)}$ however, our study data reveal pure-fat myringoplasty to be sufficient for small TM perforations. These findings are in agreement with those of previous studies comparing surgical outcomes between pure-fat myringoplasty and fat myringoplasty with $\mathrm{HA}^{25)}$ as well as those solely reporting outcomes associated with pure-fat myringoplasty. ${ }^{10,21-23,25-27)} \mathrm{PRP}$ is an autologous blood product that includes many concentrated autologous growth factors and essential proteins for tissue regeneration; therefore, some researchers have believed that PRP has the potential to not only promote healing of TM perforations and prevent TM dehydration but also stimulate centripetal migration of the outer epithelial layer. ${ }^{13,20)}$ With these characteristics of PRP, some researchers have documented that fat myringoplasty with PRP allows TM closure success rates exceeding $85 \%,{ }^{13,14)}$ reporting higher success rate than those associated with pure-fat myringoplasty. ${ }^{20)}$

Outcomes of pure-fat myringoplasty and fat myringoplasty with additional substances reported in the literature of the last 10 years are summarized in Tables 3 and 4 .

Table 3 shows success rates of $42.9 \%$ to $100 \%$ associated with pure-fat myringoplasty. In every report except that of Saliba, et al., ${ }^{2)}$ pure-fat myringoplasty was associated with postoperative hearing gains or, at least, no worsening of the ABG. Meanwhile, Table 4 shows higher success rates $(80 \%-$ 


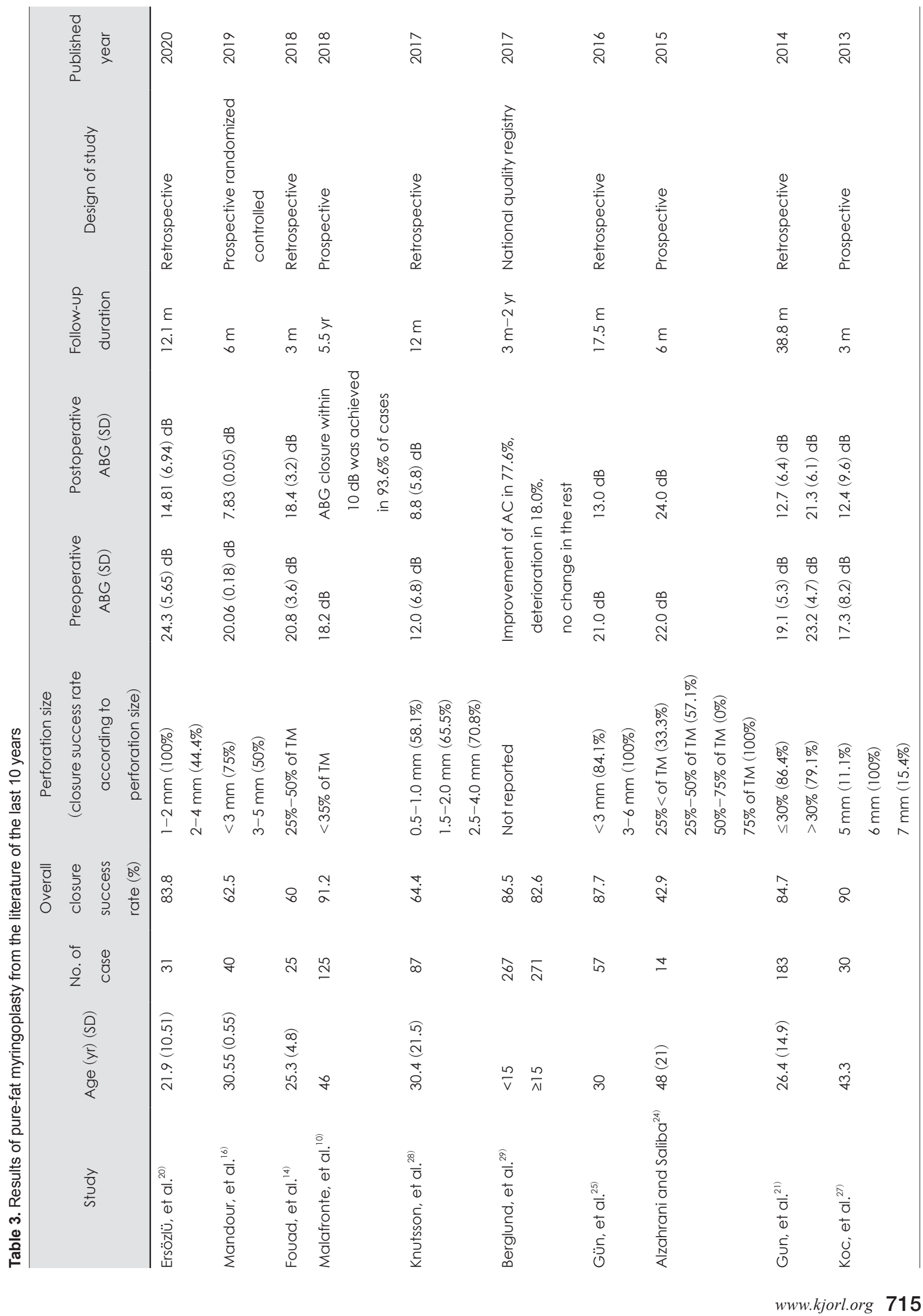




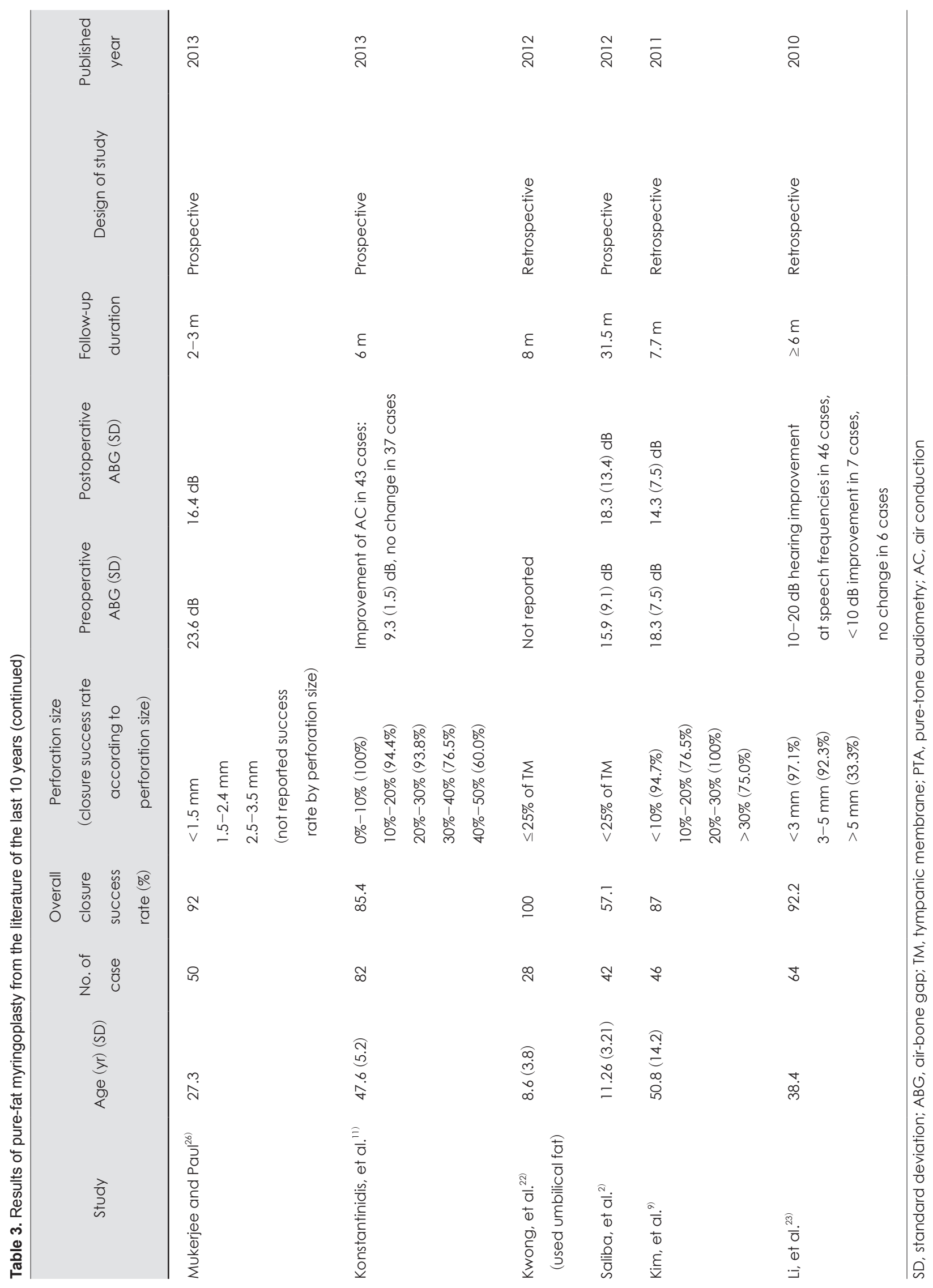


Outcomes of Fat Myringoplasty I Lee MK, et al.

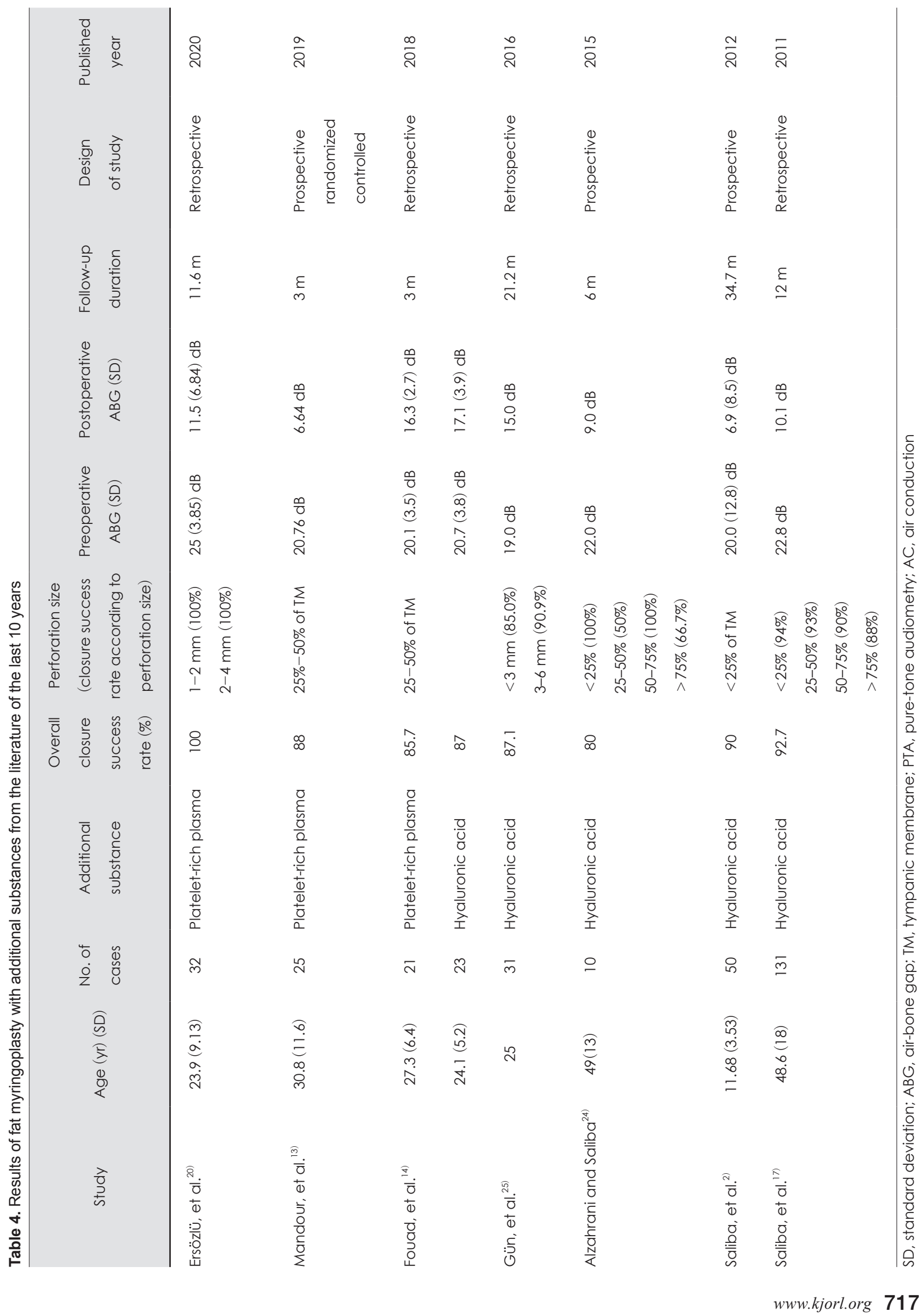


$100 \%$ ) associated with fat myringoplasty with additional substances, such as HA and PRP. However, in studies limited to small perforations, the success rates associated with pure-fat myringoplasty have been comparable to those associated with fat myringoplasty with additional substances. ${ }^{2,17,20,24,25)}$

Applying additional substances can be helpful for achieving better TM closure success rate. However, the benefit of additional substances is unclear in the context of small perforations. Pure-fat myringoplasty for small perforations was associated with a TM closure rate $>90 \%$ in our study and has been associated with success rates $>80 \%$ in articles published in the last 10 years. These outcomes are not much different from those associated with fat myringoplasty with additional substances. Furthermore, the use of additional substances inevitably not only lengthens operation times and requires additional surgical procedures, but it also increases operation costs. Therefore, pure-fat myringoplasty should be considered for patients with small TM perforations.

In audiologic results, most studies of pure-fat myringoplasty including our study, reported decreased postoperative ABG, and all studies of fat myringoplasty with additional substances in our literature review reported diminished postoperative ABG findings. Only one study reported that improved ABG results associated with pure-fat myringoplasty compared with those associated with fat myringoplasty with HA. ${ }^{2}$ In RRP studies, there was no statistically significant difference in hearing gain when comparing pure fat myringoplasty and fat myringoplasty with PRP. ${ }^{20)}$ Since our study was a retrospective study, direct comparison of whether additional substances were required for fat myringoplasty for small TM perforations was difficult. Additionally, the follow-up period was relatively short.

However, we can confirm that the results of pure-fat myringoplasty are comparable with those of fat myringoplasty with additional substances for small TM perforations based on the literature review and our results.

In conclusion, our study revealed a high closure success rate and good hearing outcomes associated with pure-fat myringoplasty for small perforations. We believe that pure fat myringoplasty is a suitable procedure for small TM perforations. However, further studies will be required to directly compare pure-fat myringoplasty and fat myringoplasty with additional substances specifically for small perforations. Moreover, applying additional substances could be helpful for achieving better closure success rates for medium-sized or larger perforations.

\section{Acknowledgments}

This work was supported by the Korea University Grants and the National Research Foundation of Korea (NRF) grant funded by the Korea government (MSIT) (No. 2020R1F1A1069424).

\section{Author Contribution}

Conceptualization: June Choi. Data curation: Min Kyu Lee, Tae Min Kim, Sung Jin Lim, Dong Heun Park, June Choi. Formal analysis: Min Kyu Lee, Tae Min Kim. Funding acquisition: Yoon Chan Rah, June Choi. Investigation: Min Kyu Lee, Tae Min Kim. Methodology: Sung Jin Lim, Dong Heun Park. Software: Sung Jin Lim, Dong Heun Park. Validation: Yoon Chan Rah, June Choi. Visualization: Min Kyu Lee, Tae Min Kim. Writing — original draft: Min Kyu Lee, June Choi. Writing — review \& editing: Min Kyu Lee, June Choi.

\section{ORCIDs}

June Choi

Min Kyu Lee

https://orcid.org/0000-0002-6330-279X

Tae Min Kim https://orcid.org/0000-0003-3447-4936

Sung Jin Lim https://orcid.org/0000-0003-3484-1660

Dong Heun Park https://orcid.org/0000-0002-5885-061X https://orcid.org/0000-0002-9671-7393

Yoon Chan Rah

\section{REFERENCES}

1) Fiorino F, Barbieri F. Fat graft myringoplasty after unsuccessful tympanic membrane repair. Eur Arch Otorhinolaryngol 2007; 264(10):1125-8.

2) Saliba I, Knapik M, Froehlich P, Abela A. Advantages of hyaluronic acid fat graft myringoplasty over fat graft myringoplasty. Arch Otolaryngol Head Neck Surg 2012;138(10):950-5.

3) Ayache S, Braccini F, Facon F, Thomassin JM. Adipose graft: An original option in myringoplasty. Otol Neurotol 2003;24(2):158-64.

4) Nishimura T, Hashimoto H, Nakanishi I, Furukawa M. Microvascular angiogenesis and apoptosis in the survival of free fat grafts. Laryngoscope 2000;110(8):1333-8.

5) Crandall DL, Hausman GJ, Kral JG. A review of the microcirculation of adipose tissue: Anatomic, metabolic, and angiogenic perspectives. Microcirculation 1997;4(2):211-32.

6) Silverman KJ, Lund DP, Zetter BR, Lainey LL, Shahood JA, Freiman DG, et al. Angiogenic activity of adipose tissue. Biochem Biophys Res Commun 1988;153(1):347-52.

7) Zhang QX, Magovern CJ, Mack CA, Budenbender KT, Ko W, Rosengart TK. Vascular endothelial growth factor is the major angiogenic factor in omentum: Mechanism of the omentummediated angiogenesis. J Surg Res 1997;67(2):147-54.

8) Ringenberg JC. Fat graft tympanoplasty. Laryngoscope 1962;72(2): 188-92.

9) Kim DK, Park SN, Yeo SW, Kim EH, Kim JE, Kim BY, et al. Clinical efficacy of fat-graft myringoplasty for perforations of different sizes and locations. Acta Otolaryngol 2011;131(1):22-6.

10) Malafronte G, Filosa B. One hundred twenty-five fat myringoplasties: Does marginal perforation matter? Clin Otolaryngol 2018;43(1): $362-5$.

11) Konstantinidis I, Malliari H, Tsakiropoulou E, Constantinidis J. Fat myringoplasty outcome analysis with otoendoscopy: Who is the suitable patient? Otol Neurotol 2013;34(1):95-9.

12) Chalishazar U. Fat plug myringoplasty. Indian J Otolaryngol Head Neck Surg 2005;57(1):43-4.

13) Mandour MF, Elsheikh MN, Khalil MF. Platelet-rich plasma fat graft versus cartilage perichondrium for repair of medium-size 
tympanic membrane perforations. Otolaryngol Head Neck Surg 2019;160(1):116-21.

14) Fouad YA, Abdelhady M, El-Anwar M, Merwad E. Topical platelet rich plasma versus hyaluronic acid during fat graft myringoplasty. Am J Otolaryngol 2018;39(6):741-5.

15) Saliba I. Hyaluronic acid fat graft myringoplasty: How we do it. Clin Otolaryngol 2008;33(6):610-4.

16) Mandour YMH, Mohammed S, Menem MOA. Bacterial cellulose graft versus fat graft in closure of tympanic membrane perforation. Am J Otolaryngol 2019;40(2):168-72.

17) Saliba I, Woods O. Hyaluronic acid fat graft myringoplasty: A minimally invasive technique. Laryngoscope 2011;121(2):375-80.

18) Güneri EA, Tekin S, Yilmaz O, Ozkara E, Erdağ TK, Ikiz AO, et al. The effects of hyaluronic acid, epidermal growth factor, and mitomycin in an experimental model of acute traumatic tympanic membrane perforation. Otol Neurotol 2003;24(3):371-6.

19) Deddens AE, Muntz HR, Lusk RP. Adipose myringoplasty in children. Laryngoscope 1993;103(2):216-9.

20) Ersözlü T, Gultekin E. A comparison of the autologous platelet-rich plasma gel fat graft myringoplasty and the fat graft myringoplasty for the closure of different sizes of tympanic membrane perforations. Ear Nose Throat J 2020;99(5):331-6.

21) Gun T, Sozen T, Boztepe OF, Gur OE, Muluk NB, Cingi C. Influence of size and site of perforation on fat graft myringoplasty. Auris Nasus Larynx 2014;41(6):507-12.

22) Kwong KM, Smith MM, Coticchia JM. Fat graft myringoplasty using umbilical fat. Int J Pediatr Otorhinolaryngol 2012;76(8):1098101.

23) Li P, Yang QT, Li YQ, Liu W, Wang T, Li Y. The selection and strategy in otoendoscopic myringoplasty with autogenous adipose tissue. Indian J Otolaryngol Head Neck Surg 2010;62(1):25-8.

24) Alzahrani M, Saliba I. Hyaluronic acid fat graft myringoplasty vs fat patch fat graft myringoplasty. Eur Arch Otorhinolaryngol 2015; 272(8):1873-7.

25) Gün T, Boztepe OF, Atan D, İkincioğulları A, Dere H. Comparison of hyaluronic acid fat graft myringoplasty, fat graft myringoplasty and temporal fascia techniques for the closure of different sizes and sites of tympanic membrane perforations. J Int Adv Otol 2016; 12(2):137-41.

26) Mukherjee M, Paul R. Minimyringoplasty: Repair of small central perforation of tympanic membrane by fat graft: A prospective study. Indian J Otolaryngol Head Neck Surg 2013;65(4):302-4

27) Koc S, Akyuz S, Gurbuzler L, Aksakal C. Fat graft myringoplasty with the newly developed surgical technique for chronic tympanic membrane perforation. Eur Arch Otorhinolaryngol 2013;270(5): 1629-33.

28) Knutsson J, Kahlin A, von Unge M. Clinical and audiological short-term and long-term outcomes of fat graft myringoplasty. Acta Otolaryngol 2017;137(9):940-4.

29) Berglund M, Florentzson R, Fransson M, Hultcrantz M, Eriksson PO, Englund E, et al. Myringoplasty outcomes from the Swedish national quality registry. Laryngoscope 2017;127(10):2389-95. 